\title{
Identificación y cuantificación de residuos de plaguicidas en hortalizas de alto consumo comercializadas en mercados $y$ supermercados de Managua
}

MSc. Josseth Mhartin Díaz Domínguez Master en Gestión Ambiental

Centro para la Investigación en Recursos Acuáticos de Nicaragua, Laboratorio de Contaminantes Orgánicos. UNAN-Managua, CIRA https://orcid.org/0000-0003-1824-0777 josseth.diaz@cira.unan.edu.ni

Fecha de recepción: 18 de diciembre, 2019 / Fecha de aceptación: 29 de enero, 2020 https://doi.org/10.5377/torreon.v8i23.9532

Palabras clave: codex alimentarius, límites máximos de residuos, matrices vegetales, plaguicidas

\section{RESUMEN}

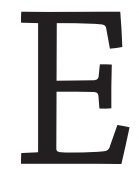
1 uso de plaguicidas para el control de plagas es una práctica común en los sistemas de producción agrícola de Nicaragua. En este estudio se determinaron las concentraciones de 21 plaguicidas (7 insecticidas organofosforados, 1 insecticida organoclorados, 3 insecticidas piretroides, 7 herbicidas y 3 fungicidas) en muestras de tomates, chiltoma, lechuga y repollo colectadas en 2 mercados populares y 2 supermercados de Managua. El análisis de las muestras refleja que el 55,0\% de estas contienen plaguicidas, lográndose identificar residuos de clorpirifos, mocap, cipermetrina, clorotalonil, imazalil, diazinon, deltametrina, simazina y 2,4-D, en concentraciones entre 0,005 mg/kg hasta 5,3 mg/ kg. El 20,0 \% de las muestras contienen concentraciones de residuos que sobrepasan los valores 
establecidos en el Codex Alimentarius en las matrices lechuga (diazinon), repollo (clorpirifos y diazinon), Tomate (mocap y deltametrina).

Nota: Este fue un proyecto de tesis de Maestría en Gestión Ambiental financiado por los fondos para proyectos de investigación de la UNAN/Managua y el CIRA/UNAN-Managua. Desarrollado en el periodo 2016-2018 bajo la línea de investigación: Inocuidad de alimentos y salud humana.

\section{INTRODUCCIÓN}

Nicaragua es un país con gran actividad agrícola, el uso de agroquímicos para el control de plagas es muy común sobre todo en los sistemas de mono cultivo no tecnificado; esto conlleva un manejo adecuado de los plaguicidas que se aplican, sin embargo, en algunos casos por desconocimiento por parte del productor, puede darse un uso indiscriminado de los plaguicidas (Cuenta Reto del Milenio Nicaragua, 2007). La manipulación y aplicación de plaguicidas puede entrañar riesgos para el ser humano ya sea como usuario o consumidor de vegetales, frutas y productos tratados (Pérez, Navarro, \& Miranda, 2013).

El uso de agroquímicos en el control de plagas debe realizarse conforme a lo que establecen las normas de inocuidad, estas establecen límites máximos permisibles de residuos para que el riesgo en los consumidores sea mínimo (Pérez, Navarro, \& Miranda, 2013). Es por eso que algunas Agencias Gubernamentales Internacionales (e.g., USFDA: Administración de Alimentos y Drogas de los Estados Unidos) y Organizaciones Internacionales (e.g., Comisión del Codex Alimentarius CAC FAO/OMS: Organización de las Naciones Unidas para la Agricultura y la Alimentación/Organización Mundial de la Salud) han establecido límites máximos de residuos de plaguicidas (LMRs) en productos de origen animal y vegetal de consumo humano o animal. El principal objetivo de los LMRs es proteger la salud de los consumidores, aunque también ayudan a promover un comercio internacional justo al asegurar prácticas de comercio claras y a mejorar la coordinación de todas las normas alimentarias (CAC, 2011).

En Nicaragua, los productos agrícolas pueden acarrear residuos no deseados de plaguicidas debido a la falta de aplicación de normas que regulen y controlen su uso indiscriminado por lo que este estudio pretende identificar y cuantificar residuos de plaguicidas en vegetales de alto consumo y determinar si cumplen con los niveles máximos de residuos de plaguicidas establecidos en el Codex Alimentarius.

\section{METODOLOGÍA}

\subsection{Colecta y pretratamiento de muestra}

Las muestras se colectaron en dos mercados populares y en dos supermercados de Managua, estos sitios son visitados por personas de diferentes distritos de esta ciudad de 
manera rutinaria para realizar sus compras. Este estudio contemplo el análisis de 40 muestras (Tabla 1) entre ellas muestras de tomates, chiltoma, lechuga y repollo, estos vegetales tienen fuerte demanda en los mercados y supermercados del país, son consumidos de manera rutinaria en la dieta de los pobladores siendo esta una razón de importancia para determinar la presencia de residuos de plaguicidas en estos alimentos.

Tabla 1. Sitios de colecta de muestras

\begin{tabular}{|l|l|l|l|l|l|}
\hline \multirow{2}{*}{$\begin{array}{c}\text { Sitio de colecta de } \\
\text { la muestra }\end{array}$} & \multicolumn{4}{c|}{ Número de muestras } & \multirow{2}{*}{ Total } \\
\cline { 2 - 5 } & Tomate & Chiltoma & Repollo & Lechuga & \\
\hline Mercados populares & 6 & 6 & 6 & 6 & 24 \\
\hline Supermercados & 6 & 2 & 4 & 4 & 16 \\
\hline TOTAL & 12 & 8 & 10 & 10 & 40 \\
\hline
\end{tabular}

El procedimiento de muestreo y el tamaño de cada muestra se realizó siguiendo las recomendaciones de la Norma Técnica Obligatoria Nicaragüense NTON-17002-02 (2001). Esta es una norma de procedimientos para el muestreo de productos vegetales. En el caso de las muestras de tomate, lechuga, cebolla y chiltoma se tomó $1 \mathrm{~kg}$ de producto por cada muestra o al menos 10 unidades y para el repollo se colectaron 5 unidades por muestra (NTON-17002-02, 2001). Una vez ingresadas las muestras al laboratorio se homogenizaron utilizando el método del cuarteo, la muestra se picó finamente con un cuchillo de acero inoxidable y una porción de ésta se trituró utilizando una mezcladora de laboratorio (BLENDER 31BL91) hasta obtener una mezcla homogénea.

\subsection{Extracción de las muestras}

La finalidad de un laboratorio es producir información (datos) relevantes y confiables para la toma de decisiones. Estos datos deben ser obtenidos con técnicas analíticas confiables, precisas y adecuadas para su fin (UNODC, 2012). El análisis de las muestras se realizó en el Laboratorio de Contaminantes Orgánicos del Centro para la Investigación en Recursos Acuáticos (CIRA/UNAN), el procedimiento de extracción se realizó como lo describe el método de la AOAC Internacional (AOAC 2007.01) "Residuos de plaguicidas en alimentos por extracción de acetonitrilo y partición con sulfato de magnesio". (Fig. 1) También llamado método QuEChERS (rápido, fácil, barato, efectivo, robusto y seguro) este método es utilizado de manera rutinaria para el análisis de plaguicidas en vegetales por muchos laboratorios de ensayos a nivel internacional. 


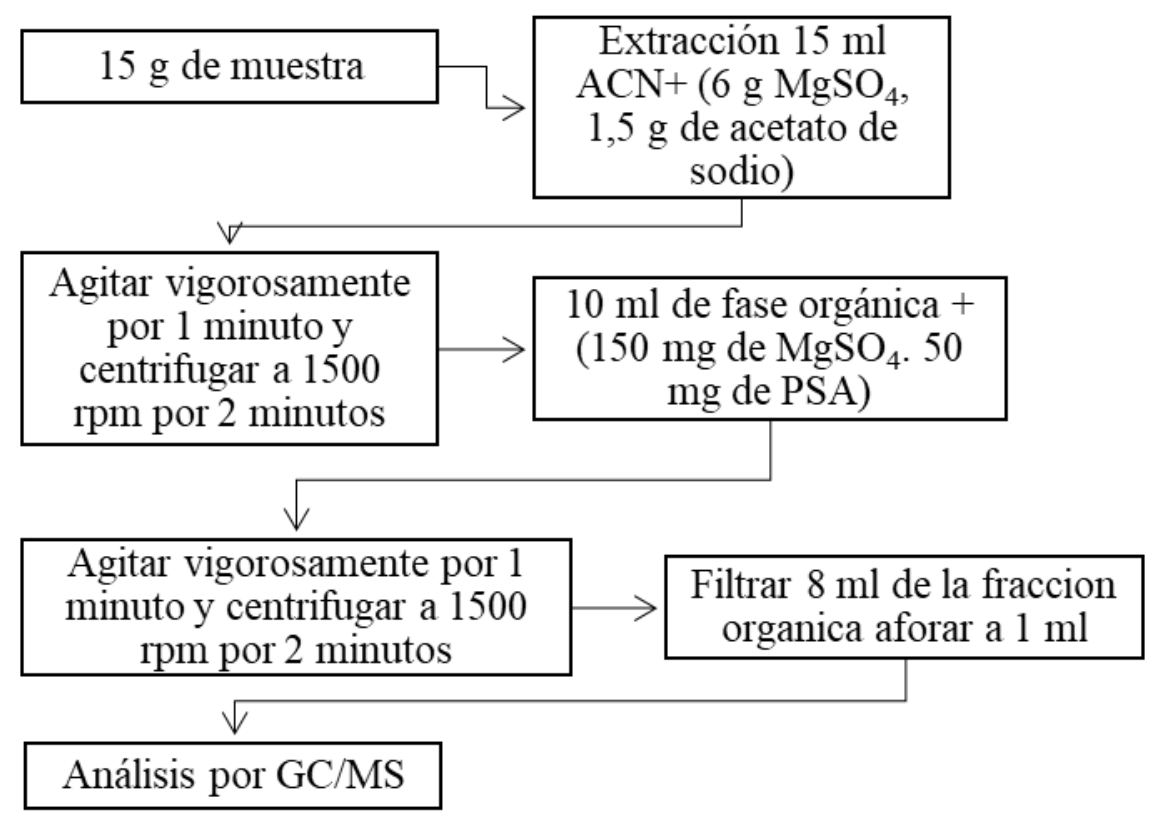

Figura 1. Extracción de plaguicidas en vegetales.

Una alícuota entre 10,0 a 15,0 gramos y 15,0 ml de acetonitrilo grado HPLC se agregan en un tubo falcon de 50,0 ml luego, se deposita el contenido total del kit de extracción QuEChERS AOAC (6,0 g de sulfato de magnesio y 1,5 g de acetato de sodio anhídrido) (Scharlau QUEXTAOAK1), se agita vigorosamente durante 1 minuto y se centrifuga (Centrifuga Thermo scientific, Sorvall ST 16) por 2 minutos a $1500 \mathrm{rpm}$.

Para la limpieza del extracto se mide una alícuota de 10,0 $\mathrm{ml}$ del extracto orgánico se deposita en el tubo Falcon que contiene kit AOAC dispersivo QuEChERS (1,20 g de sulfato de magnesio y 0,40 g de amina primaria/secundaria, PSA) (Scharlau QUDISAONK2) se agita vigorosamente durante 1 minuto y se centrifuga 2 minutos a $1500 \mathrm{rpm}$.

Se colectó una alícuota de 8,0 ml del extracto orgánico y se depositó en tubo de ensayo de $12,0 \mathrm{ml}$ y se evaporó a un volumen aproximado de $0,30 \mathrm{ml}$ con una corriente de nitrógeno inerte, luego se aforó a $1 \mathrm{ml}$ con acetonitrilo grado HPLC.

Entre los plaguicidas analizados en los extractos de cada muestra se encuentran 7 herbicidas (atrazina, pendimentalina, 2,4-D, simazina, terbutrina, ametrina), 3 funguicidas (propiconazole, clorotalonil e imazalil) y 7 insecticidas organofosforados (clorpirifos, terbufos, metamidofos, mocap (etoprofos), metil paration, malation, diazinon), 3 insecticidas piretroides (cipermetrina, trans-permetrina, deltametrina) y un plaguicida organoclorado (endofulfan). El uso de estos agroquímicos está permitido en el país para el control de distintas plagas (UNIVERSIDAD NACIONAL DE COSTA RICA, 2019), sin embargo, en algunos casos se comercializa bajo el nombre comercial y los agricultores desconocen el principio activo del mismo, (e.g., Bravonil 72 SC, principio activo clorotalonil) 


\subsection{Instrumentación y condiciones cromatográficas}

El extracto de las muestras fue inyectado en un cromatógrafo de gases (Agilent 7890A) con detector de espectrómetro de masas triple cuadrapolo (Agilent 7000B). Programación de horno $40{ }^{\circ} \mathrm{C}$, hold time 1 minuto, $40{ }^{\circ} \mathrm{C} / \mathrm{min}$ hasta $120^{\circ} \mathrm{C}, 5^{\circ} \mathrm{C} / \mathrm{min}$ hasta $240{ }^{\circ} \mathrm{C}, 12{ }^{\circ} \mathrm{C} / \mathrm{min}$ hasta $300{ }^{\circ} \mathrm{C}$, hold time 6 minutos; temperatura de inyector $250{ }^{\circ} \mathrm{C}$; volumen de inyección $1 \mu \mathrm{l}$; gas portador helio con flujo de $1 \mathrm{ml} / \mathrm{min}$. Las condiciones del espectrómetro de masas fueron: fuente de ionización impacto electrónico; voltaje de ionización $70 \mathrm{eV}$; temperatura de la fuente de ionización; línea de transferencia $280{ }^{\circ} \mathrm{C}$; Temperatura de cuadrapolo Q1 $150{ }^{\circ} \mathrm{C}, \mathrm{Q} 2150{ }^{\circ} \mathrm{C}$.

\section{RESULTADOS}

Los resultados del análisis de las muestras reflejan la presencia de 9 plaguicidas, del tipo insecticidas piretroides (cipermetrina, deltametrina); insecticidas organofosforados (mocap, diazinon); fungicidas (clorpirifos, imazalil, clorotalonil) y herbicidas (simazina, 2,4D). Las concentraciones detectadas son variables, desde $0,005 \mathrm{mg} / \mathrm{kg}$ hasta $5,3 \mathrm{mg} / \mathrm{kg}$. Algunas concentraciones sobrepasan los valores máximos de residuos que se establecen en el Codex Alimentarius.

Tabla 2. Insecticidas identificados en las muestras

\begin{tabular}{|c|c|c|c|c|}
\hline & Matriz & Código & Plaguicida & $\begin{array}{c}\text { Concentración encontrada } \\
\qquad(\mathrm{mg} / \mathrm{kg})\end{array}$ \\
\hline & \multirow{14}{*}{ Tomate } & PV-01 & mocap & 0,012 \\
\hline & & PV-02 & $2,4-\mathrm{D}$ & 0,300 \\
\hline & & PV-03 & mocap & 0,005 \\
\hline & & \multirow{3}{*}{ PV-10 } & mocap & 0,012 \\
\hline & & & cypermethrin & 0,020 \\
\hline & & & chlorothalonil & 5,300 \\
\hline & & \multirow{2}{*}{ PV-18 } & mocap & 0,002 \\
\hline & & & simazine & 0,500 \\
\hline \multirow{6}{*}{ 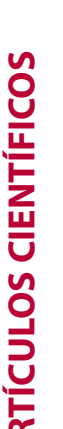 } & & PV-19 & cypermethrin & 0,010 \\
\hline & & \multirow{4}{*}{ PV-29 } & chlorpyrifos & 0,500 \\
\hline & & & deltamethrin & 0,300 \\
\hline & & & mocap & 0,020 \\
\hline & & & $2,4-D$ & 0,600 \\
\hline & & PV-30 & mocap & 0,018 \\
\hline
\end{tabular}




\begin{tabular}{|c|c|c|c|}
\hline Matriz & Código & Plaguicida & $\begin{array}{c}\text { Concentración encontrada } \\
\text { (mg/kg) }\end{array}$ \\
\hline \multirow{7}{*}{ Chiltoma } & \multirow{2}{*}{ PV-04 } & mocap & 0,050 \\
\hline & & imazalil & 0,020 \\
\hline & PV-12 & clorotalonil & 1,000 \\
\hline & PV-20 & clorpirifos & 0,500 \\
\hline & \multirow{3}{*}{ PV-33 } & clorpirifos & 0,800 \\
\hline & & cipermetrina & 0,010 \\
\hline & & clorotalonil & 0,900 \\
\hline \multirow{8}{*}{ Lechuga } & \multirow{2}{*}{ PV-08 } & clorpirifos & 0,700 \\
\hline & & diazinon & 0,300 \\
\hline & \multirow{2}{*}{ PV-16 } & cipermetrina & 0,050 \\
\hline & & diazinon & 0,600 \\
\hline & PV-27 & clorpirifos & 1,000 \\
\hline & PV-38 & cipermetrina & 0,050 \\
\hline & \multirow{2}{*}{ PV-39 } & clorpirifos & 2,000 \\
\hline & & diazinon & 0,100 \\
\hline \multirow{8}{*}{ Repollo } & \multirow{2}{*}{ PV-06 } & clorpirifos & 0,500 \\
\hline & & cipermetrina & 0,030 \\
\hline & \multirow{2}{*}{ PV-13 } & mocap & 0,100 \\
\hline & & cipermetrina & 0,150 \\
\hline & PV-14 & clorpirifos & 1,200 \\
\hline & \multirow{2}{*}{ PV-25 } & cipermetrina & 0,010 \\
\hline & & diazinon & 0,650 \\
\hline & PV-36 & cipermetrina & 0,010 \\
\hline
\end{tabular}

En el 55,0 \% de las muestras analizadas se determinaron residuos de plaguicidas, siendo las muestras de tomates las que presentan mayor cantidad de residuos, en este producto se identificó la presencia de plaguicidas en un 67,0 \% de las muestras de analizadas. 


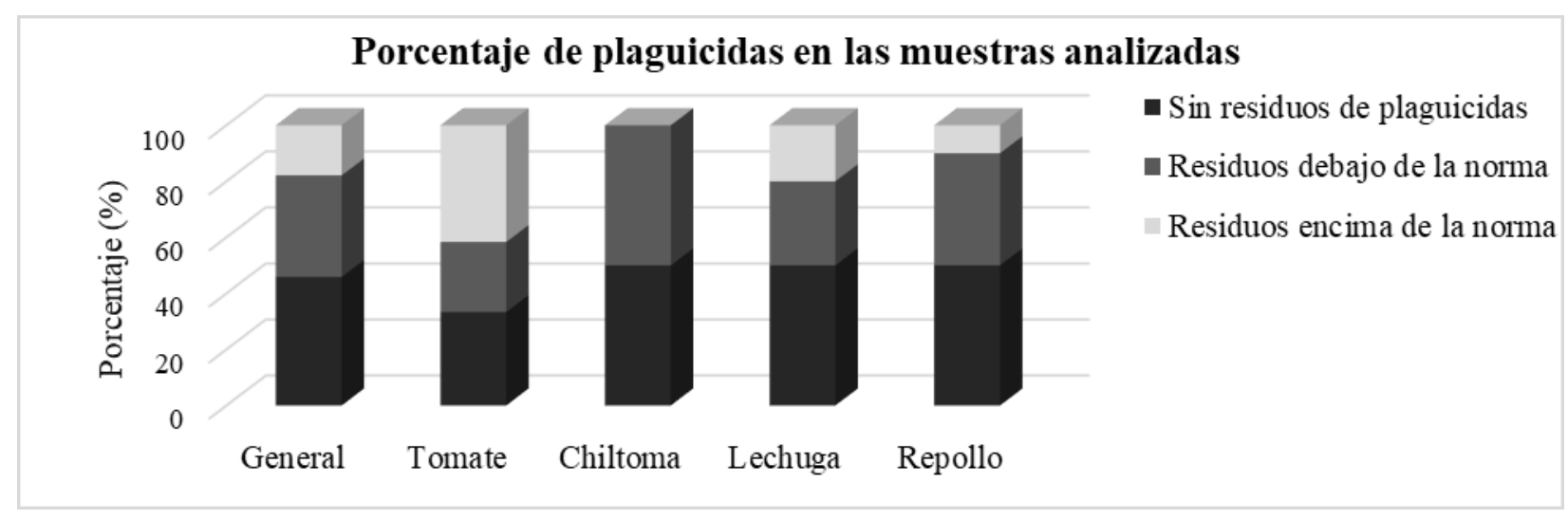

Figura 2. Porcentaje de plaguicidas encontrado en las muestras

En las muestras de chiltoma se encontraron residuos de clorpirifos, mocap, cipermetrina, clorotalonil e imazalil; En tomate se identificó cipermetrina, deltametrina, mocap, clorpirifos, clorotalonil, simazina y 2,4-D, en repollo clorpirifos, cipermetrina y diazinon, y en lechuga se encontró clorpirifos, cipermetrina y diazinon.

Muestras que sobrepasan los LMRs
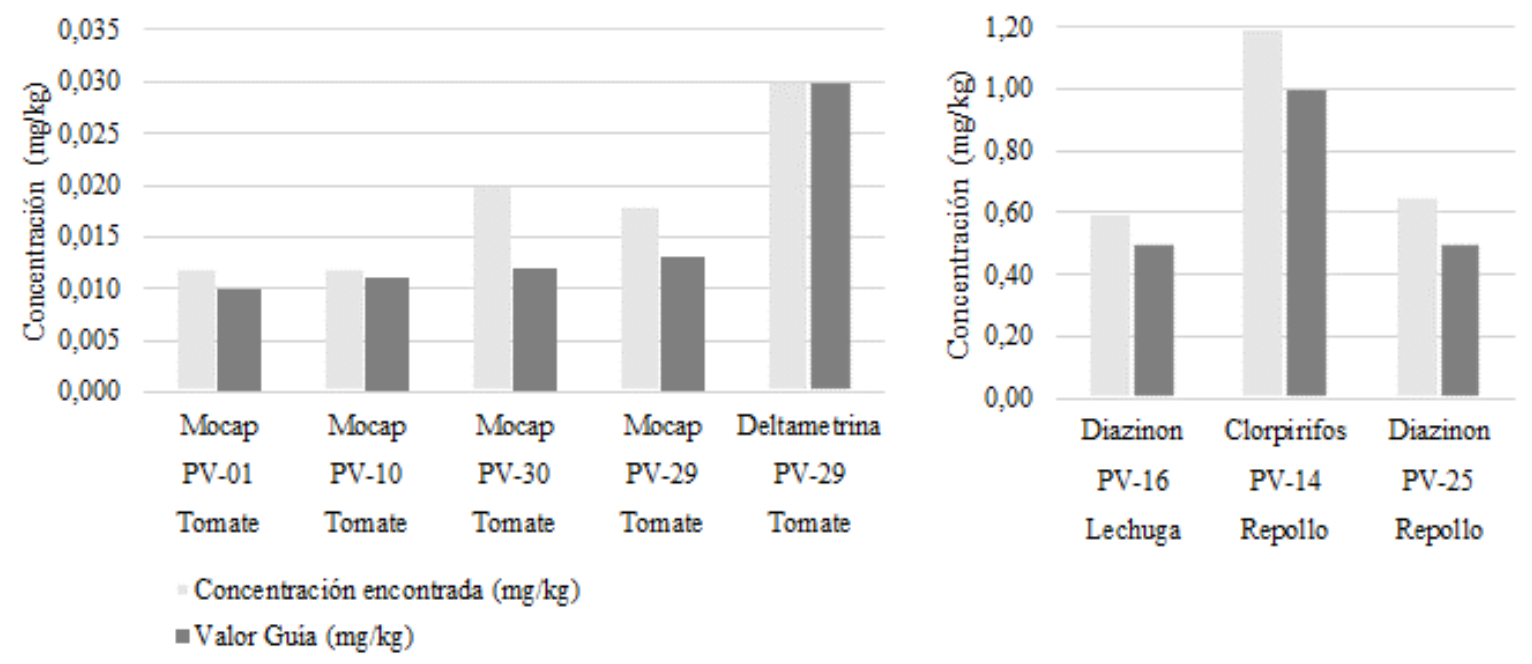

Figura 3. Plaguicidas que sobrepasan los LMRs en muestras vegetales

En muestras de lechuga, repollo y tomate se encontraron concentraciones de residuos de plaguicidas que sobrepasan los límites máximos de residuos (LMRs) establecidos por el Codex Alimentarius estimando que el 20,0\% de las muestras analizadas sobrepasan los valores guías. 


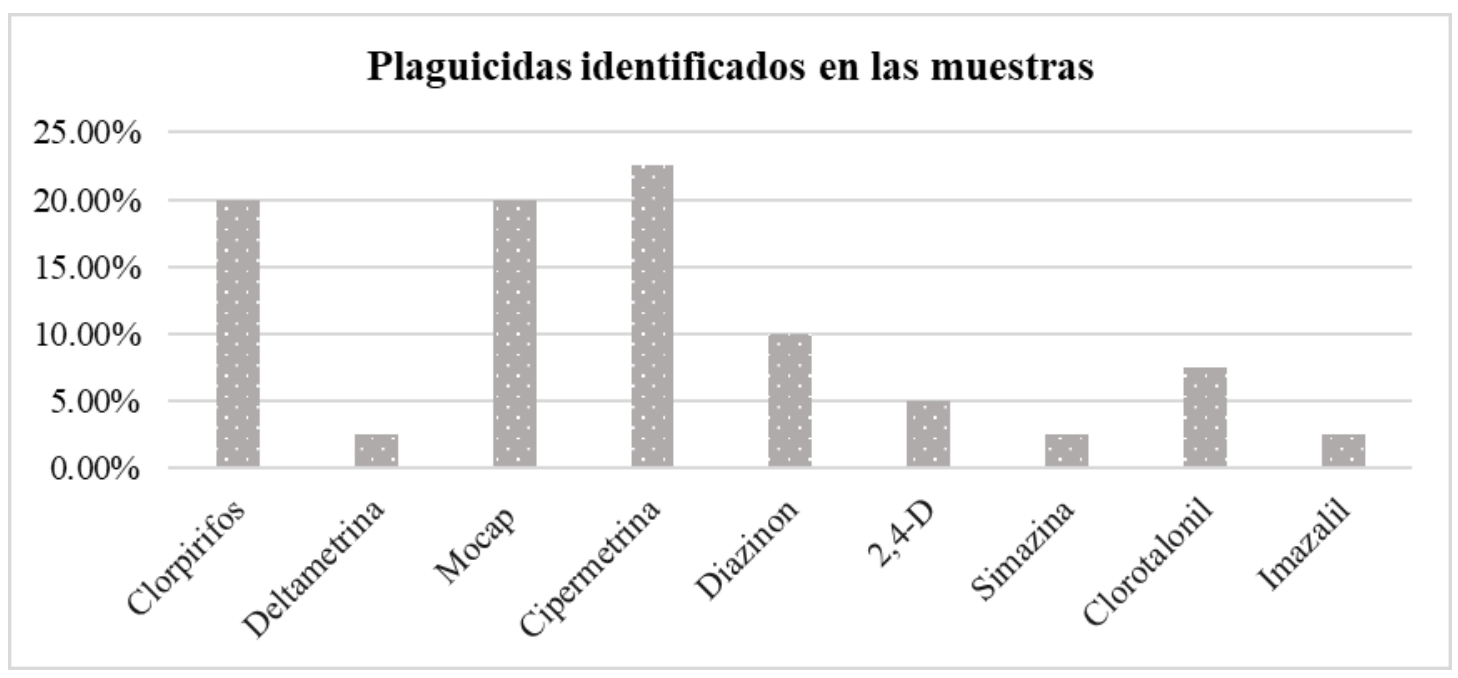

Figura 4. Plaguicidas identificados en las muestras

La cipermetrina, mocap y clorpirifos son los plaguicidas que se encuentran presentes con mayor frecuencia en las muestras en un 22,5\%, 20,0\% y 20,0\% respectivamente. La cipermetrina al igual que el clorpirifos se identificó en las todas las matrices analizadas.

\section{DISCUSIÓN}

La presencia de residuos de plaguicidas en vegetales afecta la inocuidad del producto y por ende podría producir efectos adversos en la salud del consumidor, algunos de estos compuestos poseen propiedades neurotóxicas (inhibidores de la colinesterasa), como es el caso de mocap el cual esta categorizado como IA (Extremadamente peligroso) por la Organización Mundial de la Salud (OMS) y la Universidad Nacional de Costa Rica (2019).

Este estudio refleja altas concentraciones de residuos de plaguicidas en todas las matrices vegetales estudiadas, principalmente en lechuga, repollo y tomate, en el 20,0 \% de las muestras analizadas se encontraron concentraciones de residuos de plaguicidas que sobrepasan los valores establecidos en el Codex Alimentarius. En el año 2013 el Servicio Fitosanitario del estado de Costa Rica realizó un análisis de residuos de plaguicidas en 1234 muestras de frutas, cereales, tubérculos y vegetales, colectadas en 7 provincias de Costa Rica, donde se determinó que el 6,0 $\%$ de estas sobrepasan los LMRs (Servicio fitosanitario del estado, 2013)

En tomate se identificaron residuos de mocap y deltametrina sobrepasando los LMRs. El mocap es un insecticida no sistémico clasificación IA por la OMS (extremadamente peligroso). La deltametrina es un insecticida piretroide con modo de acción no sistémico inhibe la transmisión del impulso nervioso y está clasificado como moderadamente peligroso según la OMS (UNIVERSIDAD NACIONAL DE COSTA RICA, 2019). De igual manera se identificaron residuos de clorotalonil, este fungicida también se logró encontrar en muestras analizadas por Universidad de San Carlos de Guatemala, colectadas 10 mercados de la capital de Guatemala 
en el año 2008, donde se encontraron residuos de este compuesto en el 20,0 \% de muestras de tomate analizadas (Solano, 2008).

Residuos de diazinon y clorpirifos se encontraron en repollo con concentraciones que sobrepasan los límites máximos de residuos del Codex Alimentarius. El clorpirifos en repollo también se identificó en un estudio realizado en 1998 en Nicaragua donde se analizaron 57 nuestras de esta matriz y el 24,1 \% presento residuos de este compuesto (Salgado, 1988). El clorpirifos es un insecticida no sistémico con propiedades neurotóxicas e inhibidor de la colinesterasa, posee características genotóxicas y puede producir Parkinson, además entre otros efectos crónicos está el síndrome tipo influenza, debilidad, anorexia y malestar general (Universidad nacional de Costa Rica, 2019).

En lechuga se encontraron residuos de diazinon. Este plaguicida está presente en concentraciones por encima de los valores guía de propiedades mutagénicas y teratogénicas, la ingesta diaria admisible (IDA) es de $0,002 \mathrm{mg} / \mathrm{kg}$ de peso corporal.

\section{CONCLUSIONES}

El 55 \% de las muestras analizadas presentan residuos de plaguicidas, de estas el 17,5\% sobre pasan los valores guías establecidos en el Codex Alimentarius. El 66,37 \% de las muestras analizadas de tomate presentan residuos de plaguicidas y la tercera parte de estas presentan concentraciones de plaguicidas que sobrepasan los valores guías. El fungicida clorotalonil es el agroquímico que está presente en mayores concentraciones, sin embargo, el insecticida mocap representa mayor riesgo a la salud ya que sobrepasa los valores guías en el 10,0 \% de las muestras analizadas y sobre todo en tomate tanto de los mercados populares como de los supermercados.

Los plaguicidas que se encuentran por encima de los valores guía en los productos vegetales estudiados representan un riesgo potencial a la salud debido a su elevada toxicidad. Hay que tener en cuenta que de acuerdo a este estudio los insecticidas representan un mayor riesgo ambiental y a la salud humana.

\section{BIBLIOGRAFÍA}

CAC. (2011). CAC (Codex Alimentarius Committee). Límites máximos de residuos de plaguicidas en alimentos y piensos. Obtenido de http: //www. codexalimentarius.net/pestres/data/ commodities/details.html?id=240.

Cuenta Reto del Milenio Nicaragua. (Mayo de 2007). Lineamientos y Procedimientos para el Manejo de Plagas y Enfermedades en Agricultura.

FAO. (2006). Código Internacional de conducta para la distribución y utilización de plaguicidas. ROMA: ORGANIZACIÓN DE LAS NACIONES UNIDAS PARA LA AGRICULTURA Y LA ALIMENTACIÓN. 
FAO. (2008). Food and Agriculture Organization of the the United Nations. ObtenidodeElcambioclimático,las plagas y las enfermedades transfronterizas: http://www.fao.org/tempref/docrep/ fao/010/i0142s/i0142s06.pdf

Farfán, F. (2011). Las buenas prácticas agrícolas en la caficultura. Sistemas de produccion de café en Colombia. Obtenido de https://www.cenicafe.org/es/ documents/buenasPracticasCapitulo12. pdf

GEF-REPCar, P. (2009). Monitoreo del escurrimiento de plaguicidas al caribe de Nicaragua. Managua.

Latimer, G. (2012). Official Methods of Analysis of AOAC International.

NTON-17002-02. (Diciembre de 2001). Norma de procedimiento para el muestreo de productos vegetales. Norma Técnica Obligatoria Nicarguense. Managua, Nicaragua: La Gaceta.

Pérez, M., Navarro, H., \& Miranda, E. (2013). Residuos de plaguicidas en Hortalizas: Problemática y riesgo en México. Revista Internacional de Contaminacion Ambiental, 45-64.

Salgado, T. (1988). Determinación de residuos de plaguicidas en alimentos de consumo interno. Cortés: Programa manejo de plaguicidas.
Servicio fitosanitario del estado. (2013). Informe de los residuos de plaguicidas detectados en vegetales. San José.

Solano, E. (2008). Monitoreo de plaguicidas en tomate para consumo en fresco comercializado enla ciudad de Guatemala. Ciudad de Guatemala: Universidad de San Carlos de Guatemala - Dirección General de Investigación.

UNIVERSIDAD NACIONAL DE COSTA RICA. (2019). Manual de plaguicidas de centroamérica. Obtenido de http://www. plaguicidasdecentroamerica.una.ac.cr/ index.php/base-de-datos-menu/124cipermetrina

UNODC. (2012). Oficina de las Naciones Unidas contra la Droga y el Delito. Glosario de términos sobre garantía de calidad y buenas prácticas de laboratorio. Nueva York.

Van der Hoff, G. R., \& Van Zoonen, P. (1999). Trace analysis of pesticides by gas chromatography. Journal of Chromatography, 301-322. 